\title{
A ratio chain-type exponential estimator for finite population mean using double sampling
}

\author{
Mursala Khan ${ }^{1,2^{*}}$
}

*Correspondence:

mursala.khan@yahoo.com

${ }^{1}$ Department

of Mathematics,

COMSATS Institute

of Information Technology,

Abbottabad 22060, Pakistan

Full list of author information

is available at the end of the

article

\begin{abstract}
In this article, we have proposed a ratio chain-type exponential estimator for finite population mean of the study variable under double sampling scheme using auxiliary variables. The large sample properties of the suggested strategy are derived up to first order, of approximation, and its competence conditions are carried out under which the suggested estimator is performed better than the other existing estimators discussed in the literature. An empirical study shows that the suggested strategy is more efficient than the other relevant competing estimators under two phase sampling scheme.
\end{abstract}

Keywords: Double sampling, Study variable, Bias, Auxiliary variable, Mean squared-error, Estimator, Efficiency

\section{Introduction and literature review}

To increase the precision of estimators for population mean of the study variable under double sampling design, a lot of works have been done in the field of sample survey and when the study variable is strongly connected with the auxiliary variables the precision of the estimators can be more and more. Using the knowledge of the auxiliary variables several authors have proposed different estimation technique for finite population mean of the study variable, Sukhatme (1962), have developed a general ratio-type estimator. Chand (1975), have suggested two chain ratio-type estimators to estimate the population mean using two auxiliary variables (Kiregyera 1980, 1984; Srivnstava et al. 1990; Bahl and Tuteja 1991; Srivastava 1970; Cochran 1977; Singh et al. 2006, 2007, 2011; Dash and Mishra 2011; Singh and Choudhury 2012; Khare et al. 2013; Khare and Rehman 2013) etc.

Let us consider a finite population of size $N$ of different units $U=\left\{U_{1}, U_{2}, U_{3}, \ldots, U_{N}\right\}$. Let $y$ and $x$ be the study and the auxiliary variable with corresponding values $y_{i}$ and $x_{i}$ respectively for $i$-th unit $i=\{1,2,3, \ldots, N\}$ is defined on a finite population $U$.

Let $\bar{Y}=(1 / N) \sum_{i=1}^{N} y_{i}$ and $\bar{X}=(1 / N) \sum_{i=1}^{N} x_{i}$ be the corresponding population means of the study as well as auxiliary variable respectively. Also let $S_{y}^{2}=(1 / N) \sum_{i=1}^{N}\left(y_{i}-\bar{Y}\right)^{2}$ and $S_{x}^{2}=(1 / N) \sum_{i=1}^{N}\left(x_{i}-\bar{X}\right)^{2}$ be the corresponding

(c) 2016 Khan. This article is distributed under the terms of the Creative Commons Attribution 4.0 International License (http:// creativecommons.org/licenses/by/4.0/), which permits unrestricted use, distribution, and reproduction in any medium, provided you give appropriate credit to the original author(s) and the source, provide a link to the Creative Commons license, and indicate if changes were made. 
population variances of the study as well as auxiliary variable respectively and let $C_{y}$ and $C_{x}$ be the coefficient of variation of the study as well as auxiliary variable respectively, and $\rho_{y x}$ be the correlation coefficient between $x$ and $y$.

Let $y$ and $x$ be the study and the auxiliary variable with corresponding values $y_{i}$ and $x_{i}$ respectively for $i$-th unit $i=\{1,2,3, \ldots, n\}$ in the sample. Let $\bar{y}=(1 / n) \sum_{i=1}^{n} y_{i}$ and $\bar{x}=(1 / n) \sum_{i=1}^{n} x_{i}$ be the corresponding unbiased sample means of the study as well as auxiliary variable respectively.

Also let $s_{y}^{2}=(1 / n-1) \sum_{i=1}^{n}\left(y_{i}-\bar{y}\right)^{2}$ and $s_{x}^{2}=(1 / n-1) \sum_{i=1}^{n}\left(x_{i}-\bar{x}\right)^{2}$ be the corresponding unbiased sample variances of the study as well as auxiliary variable respectively.

Let $S_{y x}, S_{y z}$ and $S_{x z}$ be the co-variances between their respective subscripts respectively. Similarly $b_{y x}=\frac{s_{y x}}{s_{x}^{2}}$ is the corresponding sample regression coefficient $y$ on $x$ based on a sample of size $n$. Also $C_{y}=\frac{S_{y}}{\bar{Y}}, C_{x}=\frac{S_{x}}{\bar{X}}$ and $C_{z}=\frac{S_{z}}{\bar{Z}}$ are the coefficients of variation of the study and auxiliary variables respectively.

The usual unbiased estimator to estimate the population mean of the study variable is

$$
\bar{y}_{0}=\frac{1}{n} \sum_{i=1}^{n} y_{i}
$$

The variance of the estimator $\bar{y}$ up to first order of approximation is, given by

$$
V\left(\bar{y}_{0}\right)=f_{1} \bar{Y}^{2} C_{y}^{2}
$$

The usual ratio and regression estimators in two phase sampling and their mean square error are, given as follows

$$
\begin{aligned}
& \bar{y}_{1}=\frac{\bar{y}}{\bar{x}} \bar{x}^{\prime} \\
& \bar{y}_{2}=\bar{y}+b_{y x}\left(\bar{x}^{\prime}-\bar{x}\right)
\end{aligned}
$$

The mean squared error and variance are given below

$$
\begin{aligned}
& \operatorname{MSE}\left(\bar{y}_{1}\right)=\bar{Y}^{2}\left[f_{1} C_{y}^{2}+f_{3}\left(C_{x}^{2}-2 \rho_{y x} C_{y} C_{x}\right)\right] \\
& \operatorname{Var}\left(\bar{y}_{2}\right)=S_{y}^{2}\left[f_{1}\left(1-\rho_{y x}^{2}\right)+f_{2} \rho_{y x}^{2}\right]
\end{aligned}
$$

where $f_{1}=\left(\frac{1}{n}-\frac{1}{N}\right), \quad f_{2}=\left(\frac{1}{n^{\prime}}-\frac{1}{N}\right)$ and $f_{3}=\left(\frac{1}{n}-\frac{1}{n^{\prime}}\right)$.

Chand (1975), proposed the following chain ratio-type estimator in double sampling by incorporating the knowledge of two auxiliary variables, the suggested estimator is, given by

$$
\bar{y}_{3}=\frac{\bar{y}}{\bar{x}} \frac{\bar{x}^{\prime}}{\bar{z}^{\prime}} \bar{Z}
$$

The mean square error of the suggested estimator is, given as 


$$
\operatorname{MSE}\left(\bar{y}_{3}\right)=\bar{Y}^{2}\left[\begin{array}{l}
f_{1} C_{y}^{2}+f_{3}\left(C_{x}^{2}-2 \rho_{y x} C_{y} C_{x}\right) \\
+f_{2}\left(C_{z}^{2}-2 \rho_{y z} C_{y} C_{z}\right)
\end{array}\right]
$$

Kiregyera (1984), suggested the following chain-type exponential estimators in two phase sampling, the suggested estimators are given as

$$
\begin{aligned}
& \bar{y}_{4}=\frac{\bar{y}}{\bar{x}}\left[\bar{x}^{\prime}+b_{x z}\left(\bar{Z}-\bar{z}^{\prime}\right)\right] \\
& \bar{y}_{5}=\bar{y}+b_{y x}\left[\left(\bar{x}^{\prime}-\bar{x}\right)-b_{x z}\left(\bar{Z}-\bar{z}^{\prime}\right)\right]
\end{aligned}
$$

The mean square errors of the suggested estimators, up to first order of approximation are, given as follows

$$
\begin{aligned}
& \operatorname{MSE}\left(\bar{y}_{4}\right)=\bar{Y}^{2}\left[\begin{array}{l}
f_{1} C_{y}^{2}+f_{3} C_{x}\left(C_{x}-2 \rho_{y x} C_{y}\right) \\
+f_{2} \rho_{x z} C_{x}\left(\rho_{x z} C_{x}-2 \rho_{y z} C_{y}\right)
\end{array}\right] \\
& \operatorname{MSE}\left(\bar{y}_{5}\right)=\bar{Y}^{2} C_{y}^{2}\left[\begin{array}{l}
f_{2} \rho_{y x} \rho_{x z}\left(\rho_{y x} \rho_{x z}-2 \rho_{y z}\right) \\
+f_{1}-f_{3} \rho_{y x}^{2}
\end{array}\right]
\end{aligned}
$$

Searls (1964), proposed an estimation procedure for population mean using known knowledge of the coefficient of variation of the auxiliary variable

$$
\begin{aligned}
& \bar{y}^{*}=a \bar{y} \\
& \operatorname{var}\left(\bar{y}^{*}\right)=(1-B) f_{1} \bar{Y}^{2} C_{y}^{2}
\end{aligned}
$$

where $a=\left\{1+f_{1} \bar{Y}^{2} C_{y}^{2}\right\}^{-1}$ and $B=f_{1} \bar{Y}^{2} C_{y}^{2}$

Khare and Rehman (2013), have proposed improved chain type estimators for population mean using auxiliary information, the suggested estimators are given by

$$
\begin{aligned}
& \bar{y}_{6}=\frac{\bar{y}^{*}}{\bar{x}}\left[\bar{x}^{\prime}+b\left(\bar{Z}-\bar{z}^{\prime}\right)\right] \\
& \bar{y}_{7}=\bar{y}^{*}+b_{1}\left[\left(\bar{x}^{\prime}-\bar{x}\right)-b_{2}\left(\bar{Z}-\bar{z}^{\prime}\right)\right]
\end{aligned}
$$

where $b, b_{1}$ and $b_{2}$ are constants.

The mean square errors of the suggested estimators, are, given by

$$
\operatorname{MSE}\left(\bar{y}_{6}\right)=\bar{Y}^{2}\left[\begin{array}{l}
+b^{2} R^{2} f_{2} C_{z}^{2}-2 b R(1-B) f_{2} \rho_{y z} C_{y} C_{z} \\
+f_{3} C_{x}\left\{C_{x}-2(1-B) \rho_{y x} C_{y}\right\} \\
+(1-B) f_{1} C_{y}^{2}
\end{array}\right]
$$

and 


$$
\operatorname{MSE}\left(\bar{y}_{7}\right)=\left[\begin{array}{l}
b_{1} f_{3} \bar{X} C_{x}\left(b_{1} \bar{X} C_{x}-2(1-B) \bar{Y} \rho_{y x} C_{y}\right) \\
+b_{1} b_{2} \bar{Z} f_{2} C_{z}\left(b_{1} b_{2} \bar{Z} C_{z}-2 \bar{Y}(1-B) \rho_{y z} C_{y}\right) \\
+\bar{Y}^{2}(1-B) f_{1} C_{y}^{2}
\end{array}\right]
$$

where the optimum values of $b, b_{1}$ and $b_{2}$ are $b_{\text {opt }}=\frac{(1-B) C_{y z}}{R C_{z}^{2}}, b_{1 \text { opt }}=\frac{-k_{2} \pm \sqrt{k_{2}^{2}-4 k_{1} k_{3}}}{2 k_{1}}$ and $b_{2 o p t}=\frac{M}{b_{1 o p t}}$.

Also $\quad M=\frac{\bar{Y}(1-B) C_{y z}}{\bar{Z} C_{z}^{2}}, \quad k_{1}=f_{3} b_{1}^{2} \bar{X}^{2} C_{x}^{2}, \quad k_{2}=f_{3}(B-1) \bar{Y} \bar{X} b_{1} C_{y x}, \quad R=\bar{Z} / \bar{X} \quad$ and $k_{2}=M \bar{Z} f_{2} C_{z}\left\{M \bar{Z} C_{z}-\bar{Y}(1-B) \rho_{y z} C_{y}\right\}$.

Singh et al. (2013), recommended a class of exponential chain ratio-product type estimator for estimating population mean using two auxiliary variables, as

$$
\bar{y}_{7}=\bar{y}\left\{\alpha \exp \left(\frac{\bar{x}^{\prime} \frac{\bar{z}}{\bar{z}^{\prime}}-\bar{x}}{\bar{x}^{\prime} \overline{\bar{Z}} \overline{\bar{z}}^{\prime}+\bar{x}}\right)+\beta \exp \left(\frac{\bar{x}-\bar{x}^{\prime} \overline{\bar{z}}}{\overline{\bar{z}^{\prime}}} \overline{\bar{x}+\bar{x}^{\prime} \overline{\bar{Z}}}\right)\right\}
$$

where $\alpha$ and $\beta$ are suitably chosen constants, such that $\alpha+\beta=1$

The minimum mean square error of the suggested estimator is given as follows

$$
\operatorname{MSE}\left(\bar{y}_{8}\right)=\bar{Y}^{2} C_{y}^{2}\left[f_{1}-\frac{\left(\rho_{y x} f_{3} C_{x}+\rho_{y z} f_{2} C_{z}\right)^{2}}{\left(f_{3} C_{x}^{2}+f_{2} C_{z}^{2}\right)}\right]
$$

where the optimum value of $\alpha$ is $\alpha_{\text {opt }}=\frac{1}{2}+\frac{\left(\rho_{y x} x_{3} C_{x y}+\rho_{y z} f_{2} C_{z y}\right)}{\left(f_{3} C_{x}^{2}+f_{2} C_{z}^{2}\right)}$.

\section{The proposed estimator}

Let us consider a finite population $U=\left\{U_{1}, U_{2}, U_{3}, \ldots, U_{N}\right\}$ of size $N$ units. To estimate the population mean $\bar{Y}$ of the variable of interest $y$ taking values $y_{i}$, in the existence of two auxiliary variables say $x$ and $z$ taking values $x_{i}$ and $z_{i}$ respectively for the $i$ th unit $U_{i}$. We assume that there is high correlation between $y$ and $x$ as compared to the correlation between $y$ and $z$ (i.e. $\rho_{y x}>\rho_{y z}>0$ ). When the population $\bar{X}$ of the auxiliary variable $x$ is unknown, but information on the other cheaply auxiliary variable $z$ closely related to $x$ but compared to $x$ remotely to $y$, is available for all the units in a population. In such a situation we use two phase sampling. In the two phase sampling scheme a large initial sample of size $n^{\prime}\left(n^{\prime}<N\right)$ is drawn from the population $U$ by using simple random sample without replacement sampling (SRSWOR) scheme and measure $x$ and $z$ to estimate $\bar{X}$. In the second phase, we draw a sample (subsample) of size $n$ from first phase sample of size $n^{\prime}$, i.e. $\left(n<n^{\prime}\right)$ by using SRSWOR or directly from the population $U$ and observed the study variable $y$.

Under the given probability sampling, we have proposed a chain-ratio-type exponential estimator for finite population mean of the study variable $y$, given by

$$
\bar{y}_{m}=\bar{y}\left(\frac{\bar{x}^{\prime}-\bar{x}}{\bar{x}^{\prime}+\bar{x}}\right)^{k_{1}}+k_{2}\left\{\bar{x}^{\prime} \exp \left(\frac{\bar{Z}-\bar{z}^{\prime}}{\bar{Z}+\bar{z}^{\prime}}\right)-\bar{x}\right\}
$$

where $k_{1}$ and $k_{2}$ are the unknown constants, whose value is to be determined for optimality conditions. 
To obtain the properties of the proposed estimator we define the following relative error terms and their expectations.

Let $e_{0}=\frac{\bar{y}-\bar{Y}}{\bar{Y}}, e_{1}=\frac{\bar{x}-\bar{X}}{\bar{X}}, e_{1}^{\prime}=\frac{\bar{x}^{\prime}-\bar{X}}{\bar{X}}, e_{2}=\frac{\bar{z}-\bar{Z}}{\bar{Z}}$ and $e_{2}^{\prime}=\frac{\bar{z}-\bar{Z}}{\bar{Z}}$.

Such that $E\left(e_{i}\right)=E\left(e_{i}^{\prime}\right)=0$. For $i=0,1,2$.

$$
\begin{aligned}
& E\left(e_{0}^{2}\right)=f_{1} C_{y}^{2}, E\left(e_{1}^{2}\right)=f_{1} C_{x}^{2}, E\left(e_{2}^{2}\right)=f_{1} C_{z}^{2}, \\
& E\left(e_{1}^{\prime 2}\right)=E\left(e_{1} e_{1}^{\prime}\right)=f_{2} C_{x}^{2}, E\left(e_{0} e_{2}^{\prime}\right)=f_{2} C_{y z}, \\
& E\left(e_{0} e_{1}\right)=f_{1} C_{y x}, E\left(e_{0} e_{1}^{\prime}\right)=f_{2} C_{y x}, E\left(e_{0} e_{2}\right)=f_{1} C_{y z}, \\
& E\left(e_{1} e_{2}^{\prime}\right)=E\left(e_{1}^{\prime} e_{2}\right)=E\left(e_{1}^{\prime} e_{2}^{\prime}\right)=f_{2} C_{x z}, \\
& E\left(e_{2}^{\prime 2}\right)=E\left(e_{2} e_{2}^{\prime}\right)=f_{2} C_{z}^{2}, E\left(e_{1} e_{2}\right)=f_{1} C_{x z} .
\end{aligned}
$$

Equation (21) can be further simplified as given by

$$
\bar{y}_{m}=\bar{Y}\left(1+e_{0}\right)\left(\frac{e_{1}^{\prime}-e_{1}}{2+e_{1}^{\prime}+e_{1}}\right)^{K_{1}}+k_{2} \bar{X}\left\{\left(1+e_{1}^{\prime}\right)\left(1-\frac{e_{2}^{\prime}}{2}+\frac{3 e_{2}^{\prime 2}}{8}\right)-\left(1+e_{1}\right)\right\}
$$

Further simplifying, up to order one

$$
\bar{y}_{m}=\bar{Y}\left\{\begin{array}{c}
1+e_{0}+\frac{k_{1}\left(e_{1}^{\prime}-e_{1}\right)}{2}-\frac{k_{1}\left(e_{1}^{\prime}+e_{1}\right)\left(e_{1}^{\prime}-e_{1}\right)}{4} \\
+\frac{k_{1}\left(k_{1}-1\right)\left(e_{1}^{\prime}-e_{1}\right)^{2}}{4}+\frac{k_{1} e_{0}\left(e_{1}^{\prime}-e_{1}\right)}{2}
\end{array}\right\}+k_{2} \bar{X}\left(e_{1}^{\prime}-\frac{e_{2}^{\prime}}{2}-\frac{e_{1}^{\prime} e_{2}^{\prime}}{2}+\frac{3 e_{2}^{\prime 2}}{8}\right)
$$

Expanding the right hand side of the Eq. (23), up to first order of approximation and subtracting $\bar{Y}$ from both sides we get

$$
\bar{y}_{m}-\bar{Y}=\bar{Y}\left\{\begin{array}{c}
e_{0}+\frac{k_{1}\left(e_{1}^{\prime}-e_{1}\right)}{2}-\frac{k_{1}\left(e_{1}^{\prime}+e_{1}\right)\left(e_{1}^{\prime}-e_{1}\right)}{4} \\
+\frac{k_{1}\left(k_{1}-1\right)\left(e_{1}^{\prime}-e_{1}\right)^{2}}{4}+\frac{k_{1} e_{0}\left(e_{1}^{\prime}-e_{1}\right)}{2}
\end{array}\right\}+k_{2} \bar{X}\left(e_{1}^{\prime}-\frac{e_{2}^{\prime}}{2}-\frac{e_{1}^{\prime} e_{2}^{\prime}}{2}+\frac{3 e_{2}^{\prime 2}}{8}\right)
$$

On squaring and taking expectation on both sides of (24), we get mean square error up to first order of approximation, given as

$$
\operatorname{MSE}\left(\bar{y}_{m}\right)=\bar{Y}^{2} C_{y}^{2}\left[\begin{array}{l}
\bar{Y}^{2} f_{1} C_{y}^{2}-\bar{Y}^{2} k_{1} f_{3} C_{y x}+\bar{Y} \bar{X} k_{2} f_{2}\left(2 C_{y x}-C_{y z}\right) \\
+\frac{1}{4} k_{2}^{2} \bar{X}^{2} f_{2}\left(C_{z}^{2}+4 C_{x}^{2}-4 C_{x z}\right)+\frac{1}{4} k_{1}^{2} \bar{Y}^{2} f_{3} C_{x}^{2}
\end{array}\right]
$$


Differentiating Eq. (25) w.r.t to $k_{1}$ and $k_{2}$ we get the optimum values of $k_{1}$ and $k_{2}$ respectively as given by, where the optimum values are $k_{1 o p t}=\frac{2 \rho_{x y} C_{y}}{C_{x}}$ and $k_{2 o p t}=\frac{-2 \bar{Y}\left(2 C_{y x}-C_{y z}\right)}{\bar{X}\left(4 C_{x}^{2}+C_{z}^{2}-4 C_{x z}\right)}$ respectively.

On substituting the optimum value of $k_{1}$ and $k_{2}$ in Eq. (25), we get the minimum mean square error of the proposed estimators, given as follows

$$
\operatorname{MSE}\left(\bar{y}_{m}\right)_{\min }=\bar{Y}^{2} C_{y}^{2}\left[f_{1}-f_{3} \rho_{x y}^{2}-\frac{f_{2}\left(2 \rho_{x y} C_{x}-\rho_{y z} C_{z}\right)^{2}}{\left(4 C_{x}\left(C_{x}-\rho_{x z} C_{z}\right)+C_{z}^{2}\right)}\right]
$$

\section{Efficiency comparison}

In this section, we have obtained some conditions by comparing the mean square errors of the estimators under which the proposed estimator performs better than the other existing estimators. The proposed estimator $\bar{y}_{m}$ is more efficient if the given conditions are satisfied.

(i) By (26) and (2), $\operatorname{MSE}\left(\bar{y}_{m}\right)_{\min } \leq \operatorname{MSE}\left(\bar{y}_{0}\right)$ if,

$$
\left[f_{3} \rho_{x y}^{2}+\frac{f_{2}\left(2 \rho_{y x} C_{x}-\rho_{y z} C_{z}\right)^{2}}{\left(4 C_{x}^{2}+C_{z}^{2}-4 C_{x z}\right)}\right] \geq 0 .
$$

(ii) By (26) and (5), $\operatorname{MSE}\left(\bar{y}_{m}\right)_{\min } \leq \operatorname{MSE}\left(\bar{y}_{1}\right)$ if,

$$
\left[\begin{array}{c}
\left(C_{x}^{2}+\rho_{x y}^{2} C_{y}^{2}-2 \rho_{y x} C_{y} C_{x}\right) \\
+\frac{C_{y}^{2} f_{2}\left(2 \rho_{x y} C_{x}-\rho_{y z} C_{z}\right)^{2}}{\left(4 C_{x}\left(C_{x}-\rho_{x z} C_{z}\right)+C_{z}^{2}\right)}
\end{array}\right] \geq 0
$$

(iii) By (26) and (6), $\operatorname{MSE}\left(\bar{y}_{m}\right)_{\min } \leq \operatorname{Var}\left(\bar{y}_{2}\right)$ if,

$$
\left[\frac{f_{2}\left(2 \rho_{y x} C_{x}-\rho_{y z} C_{z}\right)^{2}}{\left(4 C_{x}^{2}+C_{z}^{2}-4 C_{x z}\right)}\right] \geq 0 .
$$

(iv) By (26) and (8), $\operatorname{MSE}\left(\bar{y}_{m}\right)_{\min } \leq \operatorname{MSE}\left(\bar{y}_{3}\right)$ if,

$$
\left[\begin{array}{l}
f_{3}\left(C_{y}^{2} \rho_{x y}^{2}+C_{x}^{2}-2 C_{y x}\right) \\
+f_{2}\left(\frac{\left(2 C_{x y}-C_{y z}\right)^{2}}{\left(4 C_{x}^{2}+C_{z}^{2}-4 C_{x z}\right)}+\left(C_{z}^{2}-2 C_{y z}\right)\right.
\end{array}\right] \geq 0 .
$$

(v) By (26) and (11), $\operatorname{MSE}\left(\bar{y}_{m}\right)_{\min } \leq \operatorname{MSE}\left(\bar{y}_{4}\right)$ if,

$$
\left[\begin{array}{l}
f_{3}\left(C_{y}^{2} \rho_{x y}^{2}+C_{x}^{2}-2 C_{y x}\right) \\
+f_{2}\left(\begin{array}{c}
\frac{\left(2 C_{x y}-C_{y z}\right)^{2}}{\left(4 C_{x}^{2}+C_{z}^{2}-4 C_{x z}\right)}+ \\
C_{x} \rho_{x z}\left(C_{x} \rho_{x z}-2 \rho_{y z} C_{y}\right)
\end{array}\right)
\end{array}\right] \geq 0 .
$$


(vi) By (26) and (12), $\operatorname{MSE}\left(\bar{y}_{m}\right)_{\min } \leq \operatorname{MSE}\left(\bar{y}_{5}\right)$ if,

$$
\left[\rho_{y x} \rho_{x z}\left(\rho_{y x} \rho_{x z}-2 \rho_{y z}\right)+\frac{\left(2 \rho_{x y} C_{x}-\rho_{y z} C_{z}\right)^{2}}{\left(4\left(C_{x}^{2}-C_{x z}\right)+C_{z}^{2}\right)}\right] \geq 0 .
$$

(vii) By (26) and (17), $\operatorname{MSE}\left(\bar{y}_{m}\right)_{\min } \leq \operatorname{MSE}\left(\bar{y}_{6}\right)$ if,

$$
\left[\begin{array}{c}
f_{3}\left(C_{y}^{2} \rho_{x y}^{2}+C_{x}^{2}-2(1-B) C_{y x}\right)-B f_{1} C_{y}^{2} \\
+f_{2}\left\{\begin{array}{l}
\frac{\left(2 C_{x y}-C_{y z}\right)^{2}}{\left(4 C_{x}^{2}+C_{z}^{2}-4 C_{x z}\right)} \\
+b^{2} R^{2} C_{z}^{2}-2 b R(1-B) C_{y z}
\end{array}\right\}
\end{array}\right\} \geq 0 .
$$

(viii) By (26) and (18), $\operatorname{MSE}\left(\bar{y}_{m}\right)_{\min } \leq \operatorname{MSE}\left(\bar{y}_{7}\right)$ if,

$$
\left[\begin{array}{l}
f_{3}\left\{S_{y}^{2} \rho_{x y}^{2}+b_{1}^{2} S_{x}^{2}-2(1-B) b_{1} S_{y x}\right\}-\left(f_{1} S_{y}^{2}\right)^{2} \\
+f_{2}\left\{\begin{array}{l}
\frac{\bar{Y}^{2}\left(2 C_{x y}-C_{y z}\right)^{2}}{\left(4 C_{x}^{2}+C_{z}^{2}-4 C_{x z}\right)} \\
+b_{1} b_{2}\left(b_{1} b_{2} \bar{Z} S_{z}^{2}-2(1-B) S_{y z}\right)
\end{array}\right\}
\end{array}\right] \geq 0 .
$$

(ix) By (26) and (20), $\operatorname{MSE}\left(\bar{y}_{m}\right)_{\min } \leq \operatorname{MSE}\left(\bar{y}_{8}\right)$ if,

$$
\left[\begin{array}{l}
f_{3} \rho_{x y}^{2}+\frac{f_{2}\left(2 \rho_{x y} C_{x}-\rho_{y z} C_{z}\right)^{2}}{\left(4 C_{x}\left(C_{x}-\rho_{x z} C_{z}\right)+C_{z}^{2}\right)}- \\
\frac{\left(\rho_{y x} f_{3} C_{x}+\rho_{y z} f_{2} C_{z}\right)^{2}}{\left(f_{3} C_{x}^{2}+f_{2} C_{z}^{2}\right)}
\end{array}\right] \geq 0 .
$$

\section{Numerical study}

To verify the theoretical conditions under the efficiency comparison numerically, we have taken a real data set from the literature. The description and the necessary data statistics of the data is, given below.

For percent relative efficiency we use the following formula.

$$
P R E=\left[\frac{\operatorname{Var}\left(\bar{y}_{0}\right)}{\operatorname{MSE}\left(\bar{y}_{j}\right)}\right] * 100,
$$

for $j=1,2,3,4,5,6,7,8$ and $m$.

\section{Population}

The data from the population of 100 records of resale of homes from February 15 to April 30, 1993 from the files maintained by the Albuquerque Board of realtors on selling price (\$) as a study variable $y$ square feet of living space as an auxiliary variable $x$ and annual taxes (\$) as an additional variable $z$ have been taken. The numerical values of the parameters of the population are given as.

$$
\bar{Y}=1093.41, \quad \bar{X}=1697.44, \quad \bar{Z}=801.58,
$$


Table 1 Mean square errors (MSE's) of the estimators

\begin{tabular}{lllc}
\hline Estimators & \multicolumn{2}{l}{ Mean squared errors (MSE's) } \\
\cline { 2 - 4 } & $\mathbf{n}^{\prime} \mathbf{=} \mathbf{6 0}, \mathbf{n}=\mathbf{3 0}$ & $\mathbf{n}^{\prime}=\mathbf{6 5}, \mathbf{n = 3 5}$ & $\mathbf{n}^{\prime}=\mathbf{7 0 ,} \mathbf{n = 4 0}$ \\
\hline $\bar{y}_{0}$ & 3583.66 & 2852.30 & 2303.78 \\
$\bar{y}_{1}$ & 1691.43 & 1355.15 & 1087.35 \\
$\bar{y}_{2}$ & 1690.47 & 1354.39 & 1086.73 \\
$\bar{y}_{3}$ & 1192.96 & 958.26 \\
$\bar{y}_{4}$ & 1490.62 & 1024.46 & 824.15 \\
$\bar{y}_{5}$ & 1282.01 & 1021.27 & 821.59 \\
$\bar{y}_{6}$ & 1278.03 & 1018.37 & 819.03 \\
$\bar{y}_{7}$ & 1275.16 & 1017.59 & 818.40 \\
$\bar{y}_{8}$ & 1274.17 & 1181.19 & 950.65 \\
$\bar{y}_{m}$ & 1462.31 & 921.83 & 748.56 \\
\hline
\end{tabular}

Table 2 Percent relative efficiency (PRE's) of the proposed estimator with the existing estimators

\begin{tabular}{|c|c|c|c|}
\hline \multirow[t]{2}{*}{ Estimators } & \multicolumn{3}{|c|}{ Percent relative efficiency (PRE's) } \\
\hline & $n^{\prime}=60, n=30$ & $n^{\prime}=65, n=35$ & $n^{\prime}=70, n=40$ \\
\hline $\bar{y}_{0}$ & 100.00 & 100.00 & 100.00 \\
\hline $\bar{y}_{1}$ & 211.87 & 210.48 & 211.87 \\
\hline $\bar{y}_{2}$ & 211.99 & 210.60 & 211.99 \\
\hline $\bar{y}_{3}$ & 240.41 & 239.09 & 240.41 \\
\hline $\bar{y}_{4}$ & 279.54 & 278.42 & 279.54 \\
\hline $\bar{y}_{5}$ & 280.41 & 279.29 & 280.41 \\
\hline $\bar{y}_{6}$ & 281.04 & 280.09 & 281.28 \\
\hline $\bar{y}_{7}$ & 281.26 & 280.30 & 281.50 \\
\hline $\bar{y}_{8}$ & 244.79 & 303.05 & 376.54 \\
\hline $\bar{y}_{m}$ & 314.87 & 388.31 & 478.19 \\
\hline
\end{tabular}

$$
\begin{aligned}
& C_{y}^{2}=0.1285, \quad C_{x}^{2}=0.0994, \quad C_{z}^{2}=0.1560, \\
& S_{y}=391.90, \quad S_{x}=535.01, \quad S_{z}=316.62, \\
& \rho_{y x}=0.84, \quad \rho_{y z}=0.64, \quad \rho_{x z}=0.86 .
\end{aligned}
$$

\section{Conclusion}

On the basis of mean square errors and the percent relative efficiencies of the estimators as shown in Tables 1 and 2, it has been observed that the performance of the proposed estimator is better than the other relevant existing estimators discussed in the literature of survey sampling, which reveals the usefulness of suggested method in practice and would work very well in practical surveys.

Author details

${ }^{1}$ Department of Mathematics, COMSATS Institute of Information Technology, Abbottabad 22060, Pakistan. ${ }^{2}$ Carl Duisberg Center, Jägerstraße 64, 10117 Mitte-Berlin, Germany. 
Acknowledgements

This work was written by me, and we will pay the publication charges of this Journal.

\section{Competing interests}

The author declares that there is no competing interests.

Received: 10 August 2015 Accepted: 13 January 2016

Published online: 27 January 2016

\section{References}

Bahl S, Tuteja RK (1991) Ratio and product type exponential estimator. Inf Optim Sci 12:159-163

Chand L (1975) Some ratio type estimator based on two or more auxiliary variables. Unpublished Ph.D. dissertation, Lowa State University, Ames, Lowa

Cochran WG (1977) Sampling techniques. Wiley, New-York

Dash PR, Mishra G (2011) An improved class of estimators in two-phase sampling using two auxiliary variables. Commun Stat Theory Methods 40:4347-4352

Khare BB, Rehman HU (2013) Improved chain type estimators for population mean using two auxiliary variables and double sampling scheme. Int J Appl Stat Probab 1(3):82-87

Khare BB, Srivastava U, Kumar K (2013) A generalized chain ratio in regression estimator for population mean using two auxiliary characters in sample survey. J Sci Res Banaras Hindu Univ Varanasi 57:147-153

Kiregyera B (1980) A chain ratio-type estimator in finite population mean in double sampling using two auxiliary variables. Metrika 27:217-223

Kiregyera B (1984) Regression-type estimator using two auxiliary variables and model of double sampling from finite populations. Metrika 31:215-223

Searls DT (1964) The utilization of a known coefficient of variation in the estimation procedure. J Am Stat Assoc 59:1225-1226

Singh BK, Choudhury S (2012) Exponential chain ratio and product-type estimators for finite population mean under double sampling scheme. Globel J Sci Front Res Math Decis Sci 12(6):2249-4626

Singh BK, Choudhury S, Kalita D (2013) A class of exponential chain ratio-product type estimator with two auxiliary variables under double sampling scheme. Electron J Appl Stat Anal 6(2):166-174

Singh R, Chuhan P, Swan N (2007) Families of estimators for estimating population mean using known correlation coefficient in two phase sampling. Stat Transit 8(1):89-96

Singh R, Chuhan P, Swan N, Smarandache F (2011) Improved exponential estimator for population variance using two auxiliary variables. Ital J Pure Appl Math 28:101-108

Singh HP, Singh S, Kim JM (2006) General families of chain ratio type estimators of the population mean with known coefficient of variation of the second auxiliary variable in two phase sampling. J Korean Stat Soc 35(4):377-395

Srivastava SK (1970) A two phase estimator in sampling surveys. Aust J Stat 12:23-27

Srivnstava SR, Khare BB, Srivastava SR (1990) A generalized chain ratio estimator for mean of finite population. J Indian Soc Agric Stat 42(1):108-117

Sukhatme BV (1962) Some ratio type estimators in two-phase sampling. J Am Stat Assoc 57:628-632

\section{Submit your manuscript to a SpringerOpen ${ }^{\circ}$ journal and benefit from:}

- Convenient online submission

- Rigorous peer review

- Immediate publication on acceptance

- Open access: articles freely available online

- High visibility within the field

- Retaining the copyright to your article

Submit your next manuscript at $\boldsymbol{s p r i n g e r o p e n . c o m ~}$ 